\title{
The Differential Rotation of FU Ori
}

\author{
Zhaohuan Zhu ${ }^{1}$, Catherine Espaillat ${ }^{1}$, Kenneth Hinkle ${ }^{2}$, Jesus Hernandez ${ }^{1}$, \\ Lee Hartmann $^{1}$, and Nuria Calvet ${ }^{1}$ \\ zhuzh@umich.edu, ccespa@umich.edu, hinkle@noao.edu, hernandj@umich.edu, \\ lhartm@umich.edu, ncalvet@umich.edu
}

\begin{abstract}
The emission of FU Orionis objects in outburst has been identified as arising in rapidly accreting protoplanetary disks, based on a number of observational properties. A fundamental test of the accretion disk scenario is that the differentially rotating disk spectrum should produce a variation of rotational velocity with the wavelength of observation, as spectra taken at longer wavelengths probe outer, more slowly rotating disk regions. Previous observations of FU Ori have shown smaller rotation at near-infrared $(\sim 2.2 \mu \mathrm{m})$ wavelengths than observed at optical $(\sim 0.6 \mu \mathrm{m})$ wavelengths consistent with the assumption of Keplerian rotation. Here we report a spectrum from the Phoenix instrument on Gemini South which shows that differential (slower) rotation continues to be observed out to $\sim 5 \mu \mathrm{m}$. The observed spectrum is well matched by the prediction of our accretion disk model previously constructed to match the observed spectral energy distribution and the differential rotation at wavelengths $\lesssim 2.2 \mu \mathrm{m}$. This kinematic result allows us to confirm our previous inference of a large outer radius ( $1 \mathrm{AU})$ for the rapidly accreting region of the FU Ori disk, which presents difficulties for outburst models relying purely on thermal instability. While some optical spectra have been interpreted to pose problems for the disk interpretation of FU Ori, we show that the adjustment of the maximum effective temperature of the disk model, proposed in a previous paper, greatly reduces these difficulties.
\end{abstract}

Subject headings: accretion, accretion disks — stars: formation — stars: pre-main sequence

\section{Introduction}

FU Orionis systems are a class of exceptionally luminous young stellar objects found in starforming regions (Hartmann \& Kenyon 1996). Originally identified from their very large increases in

\footnotetext{
${ }^{1}$ Department of Astronomy, University of Michigan, 500 Church St., Ann Arbor, MI 48105

${ }^{2}$ National Optical Astronomy Observatory, P.O. Box 26732, Tucson, AZ 85726
} 
optical brightness over timescales of years or less (Herbig 1977), a larger group of heavily extincted probable members of the class have been identified by their characteristic infrared spectra, which strongly differ from those of typical T Tauri stars (Reipurth \& Aspin 1997; Aspin \& Reipurth 2003; Reipurth et al. 2007). Additional support for the identification of these heavily embedded objects comes from recent high-resolution infrared spectroscopy, which indicates that many of these sources are rapidly rotating and exhibit double-peaked absorption line profiles as observed in FU Ori (Greene, Aspin, \& Reipurth 2008).

The accretion disk model for FU Ori objects (Hartmann \& Kenyon 1996, and references therein) rests fundamentally on the need to explain the peculiar spectral energy distributions (SEDs) of these objects, which are much broader than that of a single temperature blackbody or star, and which exhibit a continuously varying spectral type as a function of wavelength. The disk model naturally accounts for these properties, as observations at longer wavelengths probe increasingly cooler disk regions with later spectral types; our detailed model for FU Ori matches the SED from optical wavelengths to the mid-infrared region (Zhu et al. 2007, 2008). In addition, the disk model predicts that differential rotation should be observed, with slower rotation seen at longer wavelengths, which arise from outer disk radii, and this has been confirmed by comparing optical $(\sim 0.6 \mu \mathrm{m})$ and nearinfrared $(\sim 2.2 \mu \mathrm{m})$ spectral line profiles (Hartmann \& Kenyon 1987a,b; Kenyon, Hartmann, \& Hewett 1988).

A desirable feature in a model or theory is an ability to make predictions that can be tested observationally. The disk model predicts that the observed rotational spectral line broadening should be even smaller at $\lambda \sim 5 \mu \mathrm{m}$ (using the fundamental CO vibrational transitions) than at $2.2 \mu \mathrm{m}$ (using the first overtone $\mathrm{CO}$ vibrational lines). In this paper we present a high-resolution spectrum of FU Ori in the $5 \mu \mathrm{m}$ region which matches the predictions of the disk model. We also show that some discrepancies seen in optical spectra of FU Ori in comparison with simple disk models are alleviated by a decrease in the maximum disk temperature which we proposed in Zhu et al. (2007).

\section{Observations}

A high-resolution spectrum of FU Ori at $4.9 \mu \mathrm{m}$ was obtained at UT 01:00:29 on 2007 February 4 using the Phoenix spectrometer (Hinkle et al. 1998, 2000, 2003) on the 8-m Gemini South telescope. Observations were taken with a two-pixel slit $\left(0.17^{\prime \prime}\right)$ for a resolution of $\lambda / \delta \lambda=75,000$ over the wavelength range $4.808-5.050 \mu \mathrm{m}$. We observed FU Ori at two positions along the slit for eight 2 minute exposures. We also observed the B2 III star HR1790 for telluric line correction and took 10 flat-field and dark images.

We reduced the data using IRAF. 1 We averaged the flat-field and dark images and subtracted

\footnotetext{
${ }^{1}$ IRAF is distributed by the National Optical Astronomy Observatories, which are operated by the Association of
} 
the average dark image from the average flat-field image. This averaged, dark-subtracted flatfield image was then divided into the target spectra. Images at different positions of the slit were differenced to remove the sky and dark backgrounds. We then extracted the spectra using the IRAF apall routine and later combined and flattened the spectra using splot in IRAF. The spectrum of the hot star HR1790 was used to divide out the telluric lines from the FU Ori spectrum. Wavelength calibration was computed using telluric lines from the Arcturus atlas of Hinkle et al. (1995).

We also obtained a high-resolution spectrum of FU Ori at UT 17:59:15 on 2008 November 14 using the Magellan Inamori Kyocera Echelle (MIKE) spectrograph on the $6.5 \mathrm{~m}$ Magellan Clay telescope at Las Campanas observatory (Bernstein et al. 2003). MIKE is a double echelle spectrograph which delivers full wavelength coverage from about 3350 - $5000 \AA$ (blue side) and $4900-9500 \AA$ (red side). The data were obtained in subarcsecond seeing with a $0.7^{\prime \prime}$ slit, binning $2 \times 2$ and an exposure time of 120s. The resolutions were $\sim 40,000$ and $\sim 30,000$ for the blue and red sides respectively. The MIKE data were reduced using the MIKE Redux IDL pipeline. 2

\section{Results}

Figure 1 shows the reduced Phoenix spectrum of FU Ori. Strong telluric features mean that regions around wavenumber $2009.3 \mathrm{~cm}^{-1}$ are not usable, and small residuals from the telluric correction can be seen at wavenumbers 2010.4, 2010.9, 2011.9, and $2013.5 \mathrm{~cm}^{-1}$. The spike around wavenumber $2012.5 \mathrm{~cm}^{-1}$ is due to a bad pixel. Outside of these regions, an absorption spectrum is clearly present, with relatively broad features and substantial blending. Most of the lines are due to the P-branch of the fundamental rotational-vibrational transitions of CO.

To interpret the results, we calculated a synthetic disk spectrum using the methods described by Zhu et al. (2007). The model parameters were those used by Zhu et al. (2007) to fit the SED of FU Ori and to match the observed rotational broadening observed at optical and nearinfrared wavelengths: central star mass $\sim 0.3 \mathrm{M}_{\odot}$, mass accretion rate of the inner high $\dot{M}$ disk $\sim 2.4 \times 10^{-4} \mathrm{M}_{\odot} \mathrm{yr}^{-1}$, disk inner radius $\sim 5 R_{\odot}$, outer radius of the inner high $\dot{M}$ disk $\sim 1 \mathrm{AU}$, and the disk inclination angle $\sim 55^{\circ}$. (See Figure 8 of Zhu et al. for the fit to the $2.2 \mu \mathrm{m} \mathrm{CO}$ lines). We emphasize that we have not changed or adjusted any parameters from the Zhu et al. (2007) FU Ori model; these are predicted spectra.

The lower dotted curve in Figure 1 shows the synthetic disk spectrum observed pole-on, so that individual spectral lines can be seen without the blending that occurs due to the large rotational broadening. Comparison of the nonrotating spectrum with the observations shows that FU Ori has significantly larger line widths, consistent with rapid rotation, and unlike profiles of $\mathrm{M}$ giants and supergiants. The upper dotted curve shows the synthesized spectrum using the inclination and

Universities for Research in Astronomy, Inc., under cooperative agreement with the National Science Foundation.

${ }^{2}$ http://web.mit.edu/ burles/www/MIKE/ 
central mass used to obtain a match to the $2.2 \mu \mathrm{m} \mathrm{CO}$ line widths. The agreement between synthetic and real spectra is quite good, except near $2010.7 \mathrm{~cm}^{-1}$ where we are missing the CO 5-4 P7 line in the model. This could be due to adopting too small an oscillator strength 3 . The half-width at halfdepth (HWHD) of the lines in this spectral region $(5 \mu \mathrm{m})$ is $\sim 22 \mathrm{~km} \mathrm{~s}^{-1}$, considerably smaller than the line widths measured at $2.2 \mu \mathrm{m}$ (HWHD $\sim 36 \mathrm{~km} \mathrm{~s}^{-1}$ ) (Hartmann \& Kenyon 1987a; Hartmann, Hinkle, \& Calvet 2004; Zhu et al. 2007). This HWHD at $5 \mu \mathrm{m}$ is close to the Keplerian velocity at $0.5 \mathrm{AU}$ around $0.3 \mathrm{M}_{\odot}$ central star. All the strong lines have been identified in the pole-on model spectrum. Though some water lines are present in this spectrum, they are washed out or blended in the broadened spectrum (upper dotted curve). Only the strong CO fundamental lines can be identified with certainty.

It is also worth noting that some of the unblended line profiles exhibit evidence for doublepeaked shapes predicted by simple disk models (e.g., Hartmann \& Kenyon 1987a,b). In addition, some of the blends show sharp features (eg. $2011.2 \mathrm{~cm}^{-1}$ line) which are the result of overlapping double-peaked lines. These features naturally arise in a disk model but would not be seen in rotating star models (unless large polar spots are invoked; see below).

In Figure 2 we display a segment of the MIKE spectrum of FU Ori in the wavelength range $7030-7100 \AA$ for comparison with the synthetic Keplerian disk spectrum. We again find good agreement between model and observation, demonstrating that there has been no change in the estimated optical rotational velocity of the object between the observations in Zhu et al. 2007, which we used to set the disk parameters, and this paper. The HWHD of the optical lines in this wavelength range is $\sim 65 \pm 5 \mathrm{~km} \mathrm{~s}^{-1}$, consistent with HWHD $\sim 62 \pm 5 \mathrm{~km} \mathrm{~s}^{-1}$ measured by Petrov \& Herbig (2008; PH08).

Thus, compared with HWHD of 2 micron CO first-overtone lines $\sim 36 \pm 3 \mathrm{~km} \mathrm{~s}^{-1}$ and HWHD of 5 micron CO fundamental lines $\sim 22 \pm 2 \mathrm{~km} \mathrm{~s}^{-1}$, the differential rotation in FU Ori observed over nearly an order of magnitude in wavelength is consistent with Keplerian rotation. The slow rotation observed at $5 \mu \mathrm{m}$ implies spectral formation at radii out to $\sim 0.5 \mathrm{AU}$, in agreement with our SED modeling; this supports our conclusion in Zhu et al. (2007, 2008) that the extent of the high-accretion rate disk is larger than can be explained by pure thermal instability models for outbursts (Bell \& Lin 1994).

\section{Discussion}

The consistency of the variation of rotational velocities as observed over to $\lambda \sim 0.7-5 \mu \mathrm{m}$ with Keplerian rotation seemingly provides strong evidence for the accretion disk interpretation of FU Ori. However, PH08 recently argued that while the infrared spectrum is that of an accretion disk, the optical spectrum is produced by a rapidly-rotating star with a dark polar spot. The

\footnotetext{
${ }^{3}$ This work employed the line lists from Kurucz CD ROM-15
} 
PH08 argument against a pure disk model for a central star rests on three main points: there is no evidence for a variation of absorption line width as a function of excitation potential over the wavelength range $\lambda \sim 0.52-0.86 \mu \mathrm{m}$; there is no evidence for a variation of rotational velocity with wavelength over that wavelength range; and the observed line profiles are more "boxy" or flat-bottomed than the double-peaked profiles of the disk model.

It is important to recognize that the above-listed effects expected for a disk spectrum require not only differential rotation but a temperature gradient as well. A Keplerian disk exhibiting a constant effective temperature would not show any effect of rotational velocity with either excitation or wavelength; and the double-peaked behavior of line profiles only occurs because the outer, slowly rotating regions do not fill in the profile at line center, as these regions are too cool to emit significantly at the wavelength of observation. While the standard steady disk temperature distribution $T_{\text {eff }}^{4} \propto\left[1-\left(R_{i} / R\right)^{1 / 2}\right] R^{-3}$ is a power law at large radii, it is relatively flat at distances within about twice the inner radius $R_{i}$. Therefore, observations at long wavelengths which probe the outer disk where the temperature falls rapidly with radius will exhibit stronger rotational velocity variations and more double-peaked line profiles than observations at short wavelengths probing the inner, more nearly isothermal disk.

In Zhu et al. (2007) we were forced to use a maximum disk temperature $T_{\max }=6420 \mathrm{~K}$ to match the SED of FU Ori, which is lower than the $7200 \mathrm{~K}$ maximum temperature adopted in the model used by PH08 (which was based on the earlier model by Kenyon, Hartmann, \& Hewett 1988). Lowering the maximum temperature has the effect of making the flatter part of the accretion disk temperature distribution more dominant at optical wavelengths. As shown in Table 1 and Figure 3, this model predicts essentially no variation of line width with lower level excitation potential and a very slight dependence on wavelength in the optical region. (Note that PH08 predict a much larger effect of line width on excitation potential than Welty et al. (1992) for what should be essentially the same disk model; the reason for the large discrepancy is unclear.) In any case, measurement of rotation is best done through cross-correlation using suitable templates, as many of the lines used by $\mathrm{PH} 08$ are blends and introduce very large scatter into the model predictions (see Table 1 and Figure 3).

PH08 also noted that their disk model predicts very strong TiO absorption bands at $\sim 7054$ and $7087 \AA$ which are not observed. However, as shown in Figure 2, our lower-temperature disk model does not predict strong $\mathrm{TiO}$ absorption bands in this region. In addition there is evidence for $7087 \AA$ bandhead absorption in our MIKE spectra, at the level predicted by our disk model. Once again this difference in the predicted disk model spectra arises simply by reducing the maximum disk temperature, which increases the importance of hot inner disk relative to the outer cool disk at the wavelength of observation.

It has long been known that many optical line profiles in FU Ori are less double-peaked than predicted by simple quiescent disk models (Hartmann \& Kenyon 1985). There are, however, alternative possibilities to explain the profiles which do not demand abandonment of the accretion disk 
hypothesis. If, as currently thought, ionized disks accrete through the action of the magnetorotational instability (MRI; Balbus \& Hawley 1998), such disks must be turbulent. The disk models with resolved vertical structure computed by Miller \& Stone (2000) predict that turbulence driven by the MRI in the central layers of the disk produces waves which propagate outward and shock in the upper layers. It would be surprising if the MRI did not produce significant turbulent motions in the upper atmospheric layers of the disk, which would tend to wash out the double profile structure. Hartmann, Hinkle, \& Calvet (2004) found that that some mildly supersonic turbulence was needed to explain the ${ }^{12} \mathrm{CO}$ first-overtone lines of FU Ori.

It should be noted that the standard steady disk structure may not be completely applicable in the innermost disk. Standard thin-disk models predict that accretion onto a slowly rotating star should give rise to boundary-layer emission with roughly half the system luminosity; this is not observed in FU Ori (Kenyon et al. 1989). Popham et al. (1996) considered disk models which suppress boundary layer radiation; such models exhibit less doubled line profiles in inner disk regions, largely because the angular velocity of the disk departs from Keplerian values near the inner disk boundary.

To explain the optical spectrum of FU Ori with a central star, the star would be required to have essentially the same total system luminosity $L \sim 230 \mathrm{~L}_{\odot}$, and would need to have a radius roughly twice the inner radius of the disk model, $R \sim 10 \mathrm{R}_{\odot}$ (Zhu et al. 2007). Assuming Keplerian rotation for the infrared disk, Zhu et al. estimated a central mass $M \sim 0.3 \mathrm{M}_{\odot}$. Such a star cannot be an isolated product of stellar evolution, as it has an implausibly short Kelvin-Helmholtz contraction time $\sim G M^{2} R^{-1} L^{-1} \sim 1200 \mathrm{yr}$. The energy to power the star would have to come from disk accretion, which would also potentially explain the outburst (Larson 1983). However, as the ratio of optical-to-infrared rotational velocities is consistent with a Keplerian profile, this implies that any central star would have to be rotating nearly at breakup; this means that the assumption of solid-body rotation in the PH08 model is unlikely to be correct. It is also unclear whether the accretion of a large amount of hot disk material would add enough angular momentum to spin up the outer layers of the star to breakup velocity as it expanded the outer atmosphere.

In summary, the accretion disk model for FU Ori provides a coherent explanation of the observed spectral energy distribution and differential rotation over more than a decade in wavelength. The slow rotation observed at $5 \mu \mathrm{m}$ supports our previous result that the high mass accretion rate disk could extend to $0.5-1 \mathrm{AU}$, which is significantly larger than that predicted by the pure thermal instability theory (Bell \& Lin 1994). On the other hand, the theory incorporating both gravitational and magnetorotational (Gammie 1999; Book \& Hartmann 2005; Zhu et al. 2009) successfully predicts AU scale high mass accretion rate inner disk during outbursts (Zhu et al. 2009). With the advent of more powerful computers and sophisticated magnetohydrodynamic codes, and the assumption of MRI-driven accretion, it should be possible to explore the possibility that atmospheric turbulence and/or nonstandard inner thin disk structure can explain details of the optical line profiles. 
This work is supported in part by NASA grant NNX08AI39G and is based in part on observations obtained at the Gemini Observatory, which is operated by the Association of Universities

for Research in Astronomy, Inc., under a cooperative agreement with the NSF on behalf of the Gemini partnership: the National Science Foundation (United States), the Science and Technology Facilities Council (United Kingdom), the National Research Council (Canada), CONICYT (Chile), the Australian Research Council (Australia), Ministrio da Cincia e Tecnologia (Brazil) and Ministerio de Ciencia, Tecnologa e Innovacin Productiva (Argentina). The observations were obtained with the Phoenix infrared spectrograph, which was developed and is operated by the National Optical Astronomy Observatory. The Gemini/Phoenix spectra were obtained as part of program GS-2007A-C-4.

\section{REFERENCES}

Armitage, P. J., Livio, M., \& Pringle, J. E. 2001, MNRAS, 324, 705

Aspin, C., \& Reipurth, B. 2003, AJ, 126, 2936

Balbus, S. A., \& Hawley, J. F. 1998, Reviews of Modern Physics, 70, 1

Bell, K. R., \& Lin, D. N. C. 1994, ApJ, 427, 987

Bernstein, R., Shectman, S. A., Gunnels, S. M., Mochnacki, S., \& Athey, A. E. 2003, Proc. SPIE, 4841, 1694

Book, L. G., \& Hartmann, L. 2005, BAAS, 37, 1287

Fleming, T., \& Stone, J. M. 2003, ApJ, 585, 908

Gammie, C. F. 1996, ApJ, 457, 355

Gammie, C. F. 1999, Astrophysical Discs - an EC Summer School, 160, 122

Greene, T. P., Aspin, C., \& Reipurth, B. 2008, AJ, 135, 1421

Hartmann, L., Hinkle, K., \& Calvet, N. 2004, ApJ, 609, 906

Hartmann, L., \& Kenyon, S. J. 1985, ApJ, 299, 462

Hartmann, L., \& Kenyon, S. J. 1987a ApJ, 312, 243

Hartmann, L., \& Kenyon, S. J. 1987b, ApJ, 322, 393

Hartmann, L., \& Kenyon, S. J. 1996, ARA\&A, 34, 207

Herbig, G. H. 1977, ApJ, 217, 693

Hinkle, K., Wallace, L., \& Livingston, W. 1995, PASP, 107, 1042 
Hinkle, K. H., Cuberly, R. W., Gaughan, N. A., Heynssens, J. B., Joyce, R. R., Ridgway, S. T., Schmitt, P., \& Simmons, J. E. 1998, Proc. SPIE, 3354, 810

Hinkle, K. H., Joyce, R. R., Sharp, N., \& Valenti, J. A. 2000, Proc. SPIE, 4008, 720

Hinkle, K. H., et al. 2003, Proc. SPIE, 4834, 353

Kenyon, S. J., Hartmann, L., \& Hewett, R. 1988, ApJ, 325, 231

Kenyon, S. J., Hartmann, L., Imhoff, C. L., \& Cassatella, A. 1989, ApJ, 344, 925

Larson, R. B. 1983, Revista Mexicana de Astronomia y Astrofisica, vol. 7, 7, 219

Miller, K. A., \& Stone, J. M. 2000, ApJ, 534, 398

Petrov, P. P., \& Herbig, G. H. 2008, AJ, 136, 676

Popham, R., Kenyon, S., Hartmann, L., \& Narayan, R. 1996, ApJ, 473, 422

Reipurth, B., \& Aspin, C. 1997, AJ, 114, 2700

Reipurth, B., Hartmann, L., Kenyon, S. J., Smette, A., \& Bouchet, P. 2002, AJ, 124, 2194

Reipurth, B., Aspin, C., Beck, T., Brogan, C., Connelley, M. S., \& Herbig, G. H. 2007, AJ, 133, 1000

Welty, A. D., Strom, S. E., Edwards, S., Kenyon, S. J., \& Hartmann, L. W. 1992, ApJ, 397, 260

Zhu, Z., Hartmann, L., Calvet, N., Hernandez, J., Muzerolle, J., \& Tannirkulam, A.-K. 2007, ApJ, 669,483

Zhu, Z., Hartmann, L., Calvet, N., Hernandez, J., Tannirkulam, A.-K., \& D’Alessio, P. 2008, ApJ, 684,1281

Zhu, Z., Hartmann, L., Gammie, C., 2009, in preparation 
Table 1: HWHD of lines selected by PH08 as measured from Our synthetic disk spectrum

\begin{tabular}{|c|c|c|c|c|c|c|c|c|c|}
\hline $\begin{array}{c}\lambda \\
(\AA)\end{array}$ & Ion & $\begin{array}{c}\mathrm{EP} \\
(\mathrm{eV})\end{array}$ & $\begin{array}{l}\text { HWHD } \\
\left(\mathrm{km} \mathrm{s}^{-1}\right)\end{array}$ & Grade $^{\mathrm{a}}$ & $\begin{array}{c}\lambda \\
(\AA)\end{array}$ & Ion & $\begin{array}{c}\mathrm{EP} \\
(\mathrm{eV})\end{array}$ & $\begin{array}{c}\text { HWHD } \\
\left(\mathrm{km} \mathrm{s}^{-1}\right)\end{array}$ & Grade \\
\hline 5383.37 & Fe I & 4.31 & - & 0 & 6726.66 & Fe I & 4.61 & 66.2 & 3 \\
\hline 5717.83 & Fe I & 4.28 & - & 1 & 6767.79 & $\mathrm{Ni} \mathrm{I}$ & 1.83 & 65.4 & 3 \\
\hline 5772.15 & Si I & 5.08 & - & 0 & 6810.26 & Fe I & 4.61 & - & 1 \\
\hline 5775.08 & Fe I & 4.22 & - & 1 & 6814.94 & Co I & 1.96 & - & 0 \\
\hline 5862.35 & Fe I & 4.55 & - & 0 & 6828.59 & Fe I & 4.64 & 78 & 3 \\
\hline 5899.29 & Ti I & 1.05 & 61.3 & 2 & 7090.38 & Fe I & 4.23 & 66.8 & 4 \\
\hline 5922.11 & Ti I & 1.05 & 61.8 & 3 & 7122.19 & $\mathrm{Ni} \mathrm{I}$ & 3.54 & 66.1 & 4 \\
\hline 5934.66 & Fe I & 3.93 & 78.4 & 3 & 7344.70 & Ti I & 1.46 & 76.8 & 2 \\
\hline 5965.83 & $\mathrm{Ti} \mathrm{I}$ & 1.88 & 65.9 & 3 & 7393.60 & $\mathrm{Ni} \mathrm{I}$ & 3.61 & 68 & 3 \\
\hline 5987.07 & Fe I & 4.80 & 71.7 & 3 & 7445.75 & Fe I & 4.26 & - & 0 \\
\hline 6016.66 & Fe I & 3.55 & - & 1 & 7511.02 & Fe I & 4.18 & 61.7 & 4 \\
\hline 6024.06 & Fe I & 4.55 & - & 1 & 7525.11 & $\mathrm{Ni} \mathrm{I}$ & 3.63 & - & 1 \\
\hline 6027.05 & Fe I & 4.09 & - & 0 & 7555.60 & $\mathrm{Ni} \mathrm{I}$ & 3.85 & 68.3 & 3 \\
\hline 6056.01 & Fe I & 4.73 & - & 1 & 7568.89 & $\mathrm{Fe} I$ & 4.28 & 69.4 & 4 \\
\hline 6108.11 & $\mathrm{Ni} \mathrm{I}$ & 1.68 & - & 1 & 7574.04 & $\mathrm{Ni} \mathrm{I}$ & 3.83 & - & 1 \\
\hline 6180.20 & Fe I & 2.73 & 70 & 2 & 7586.01 & Fe I & 4.31 & 65.8 & 5 \\
\hline 6270.23 & Fe I & 2.86 & 72.2 & 3 & 7727.61 & $\mathrm{Ni} \mathrm{I}$ & 3.68 & 65.4 & 4 \\
\hline 6355.03 & Fe I & 2.85 & 64.4 & 3 & 7780.55 & Fe I & 4.47 & 65.4 & 4 \\
\hline 6358.70 & Fe I & 0.86 & 66.05 & 4 & 7788.94 & Ni I & 1.95 & 64.13 & 4 \\
\hline 6380.74 & Fe I & 4.19 & 72.2 & 3 & 7855.44 & Fe I & 5.06 & 62.1 & 4 \\
\hline 6411.65 & Fe I & 3.65 & 67.8 & 2 & 7912.87 & Fe I & 0.86 & - & 0 \\
\hline 6439.08 & $\mathrm{Ca} \mathrm{I}$ & 2.53 & 74.5 & 3 & 7937.13 & Fe I & 4.33 & 63.5 & 4 \\
\hline 6471.66 & $\mathrm{Ca} \mathrm{I}$ & 2.53 & 72.8 & 3 & 8075.15 & Fe I & 0.92 & - & 0 \\
\hline 6475.62 & Fe I & 2.56 & 66.3 & 5 & 8080.55 & Fe I & 3.30 & - & 0 \\
\hline 6569.22 & Fe I & 4.73 & 67.8 & 5 & 8085.18 & Fe I & 4.45 & 64.7 & 3 \\
\hline 6581.21 & Fe I & 1.49 & 64.7 & 4 & 8426.51 & Ti I & 0.83 & - & 0 \\
\hline 6586.31 & $\mathrm{Ni} \mathrm{I}$ & 1.95 & - & 1 & 8611.80 & Fe I & 2.85 & 65.0 & 4 \\
\hline 6707.89 & Li I & 0.00 & - & 0 & 8621.60 & Fe I & 2.95 & 63.5 & 3 \\
\hline 6717.68 & $\mathrm{Ca} \mathrm{I}$ & 2.71 & 69.0 & 4 & 8648.47 & Si I & 6.21 & 73.9 & 3 \\
\hline 6721.85 & Si I & 5.86 & 65.2 & 2 & & & & & \\
\hline
\end{tabular}

${ }^{a}$ HWHD means "half-width at half-depth" as illustrated and defined in Figure 3 of PH08. Grade indicates the amount of line blending due to rapid rotation: grades 0 and 1 signify badly blended lines, grade 2 identifies less-blended lines that are difficult to use, and grade 3 means that the line is moderately blended so that the true HWHD may be slightly smaller than our measured value to within $10 \mathrm{~km} \mathrm{~s}^{-1}$. Grade 4 means the line is slightly blended with an errorbar of only $3 \mathrm{~km} \mathrm{~s}^{-1}$, while grade 5 means that the line is blend-free. 


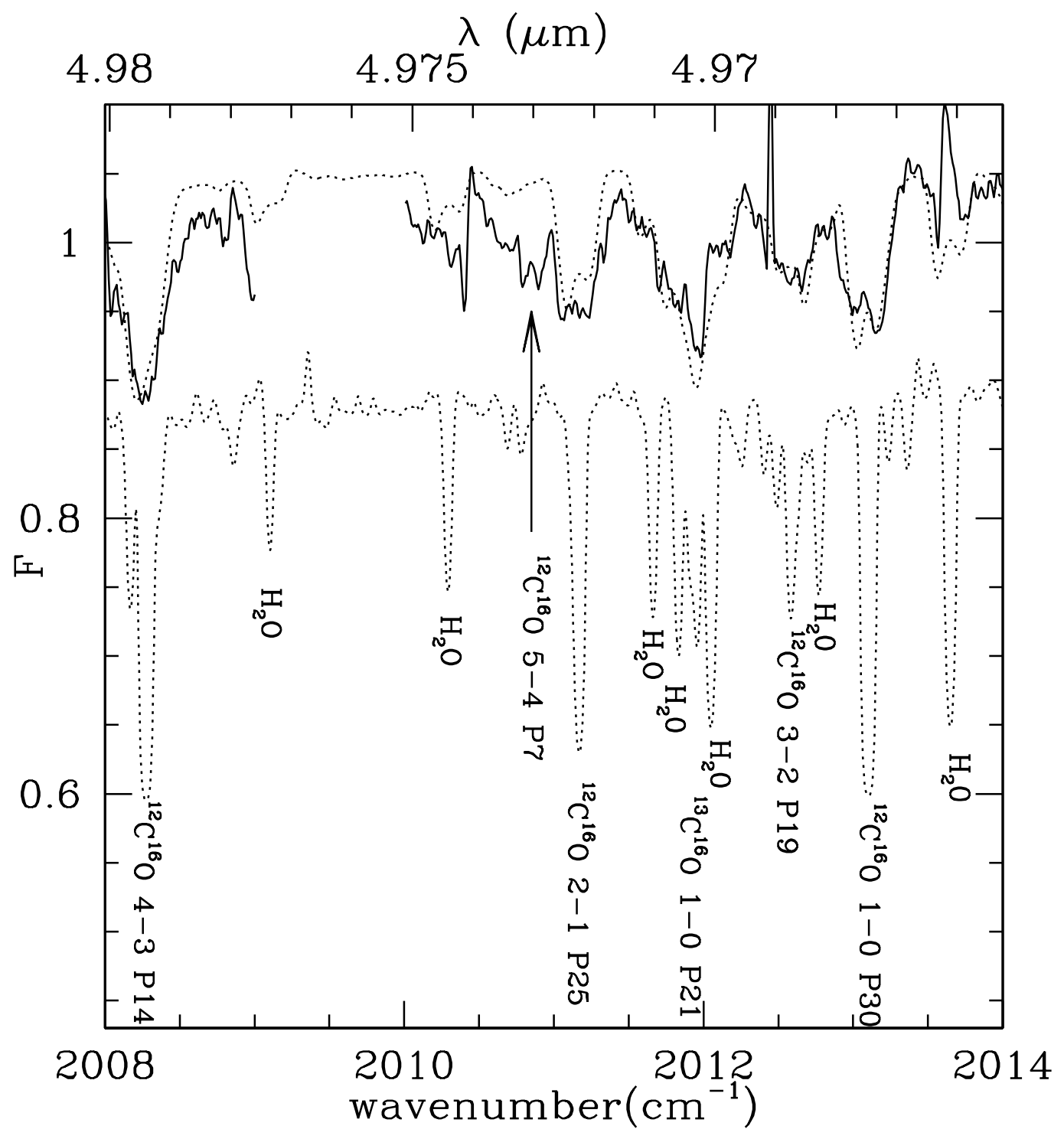

Fig. 1.- Observed $4.9 \mu \mathrm{m}$ Phoenix spectrum of FU Ori (solid curve) compared with our synthetic disk model spectrum (upper dotted curve). While the wavenumber is shown at the bottom, the wavelength in microns is shown at the top of the figure. The lower dotted curve shows the model spectrum observed pole-on, which removes the rotational broadening, illustrating the need for rapid rotation to match the observations (see the text). The strong lines are identified in the lower model spectrum. The arrow points to the strong CO line which is missing in our model. 


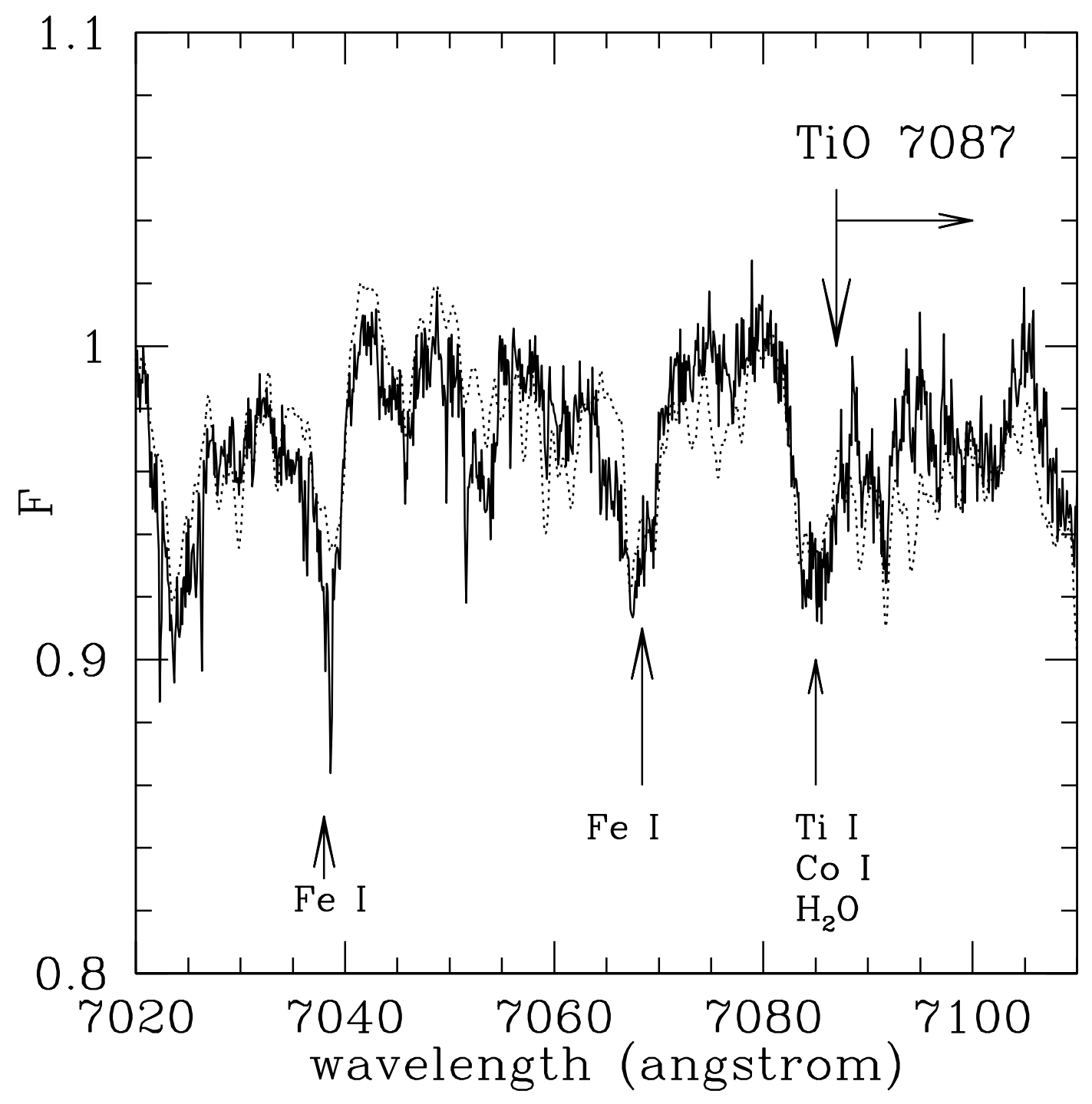

Fig. 2.- Comparison of the observed MIKE spectrum of FU Ori near $7080 \AA$ (solid curve) with the synthetic disk spectrum (dotted curve). Compared with the continuum flux $\sim 7080 \AA$, there is some evidence for weak $\mathrm{TiO} 7087 \AA$ band absorption in both the observed and the model spectrum (see text). 

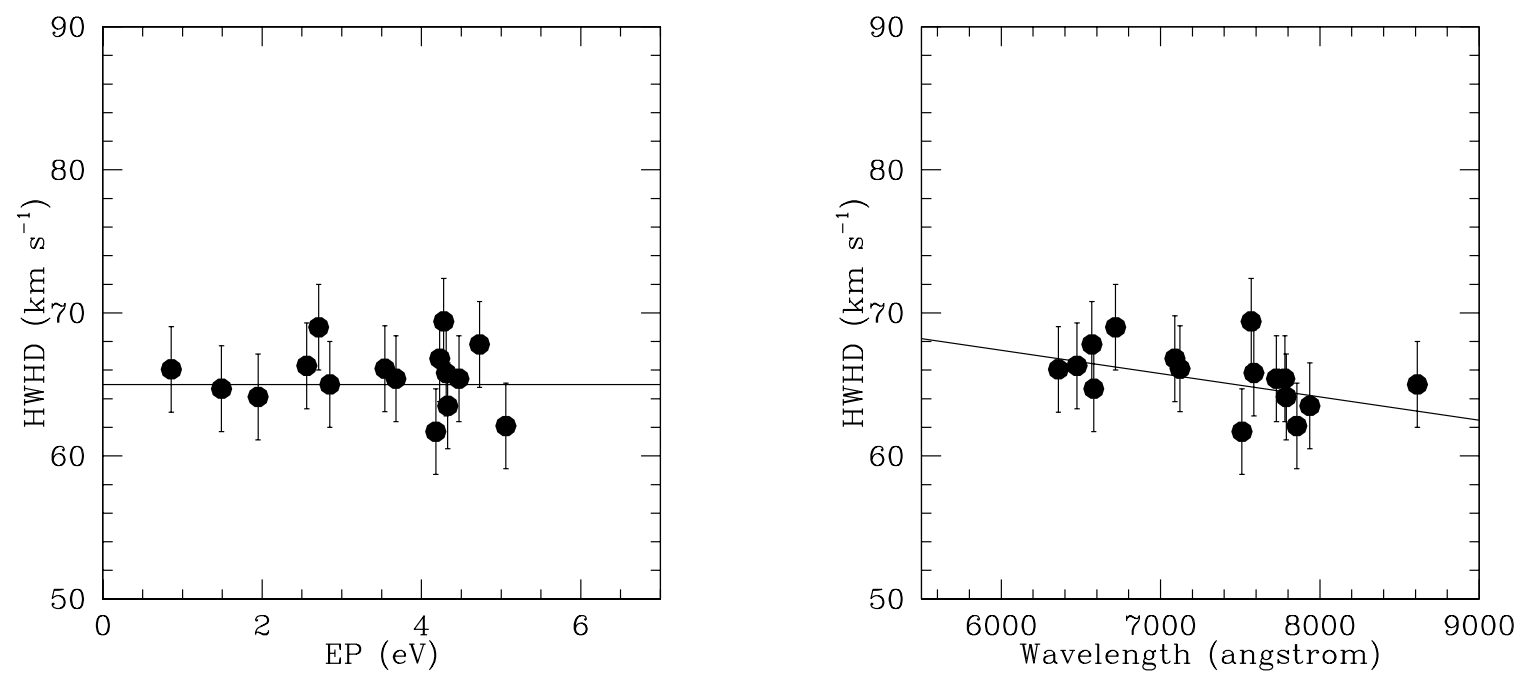

Fig. 3.- Dependence of the model line half-width at half-depth (HWHD) on excitation potential (EP) (left) and the wavelength (right), taken from Table 1. We show only the grade 4 and 5 line widths for clarity (see Table 1 for their definitions). 